\title{
ORIGINAL
}

\section{Sustained reduction of catheter-associated bloodstream infections with enhancement of catheter bundle by chlorhexidine dressings over 11 years}

\author{
Philippe Eggimann ${ }^{1,6^{*}} \mathbb{D}$, Jean-Luc Pagani ${ }^{1}$, Elise Dupuis-Lozeron² ${ }^{2}$ Bruce Ekholm MS ${ }^{3}$, \\ Marie-Josèphe Thévenin ${ }^{4}$, Christine Joseph', Jean-Pierre Revelly ${ }^{1}$ and Yok-Ai Que ${ }^{5}$
}

() 2019 The Author(s)

\begin{abstract}
Background: Prospective randomized controlled studies have demonstrated that addition of chlorhexidine (CHG) dressings reduces the rate of catheter (central venous and arterial)-associated bloodstream infections (CABSIs). However, studies confirming their impact in a real-world setting are lacking.

Methods: We conducted a real-world data study evaluating the impact of incrementally introducing chlorhexidine dressings (sponge or gel) in addition to an ongoing catheter bundle on the rates of CABSI, expressed as incidence density rates per 1000 catheter-days measured as part of a surveillance program. Poisson regression models were used to compare infection rates over time. Both dressings were used simultaneously during one of the five study periods.

Results: From 2006 to 2014, 18,286 patients were admitted (91,292 ICU-days and 155,242 catheter-days). We recorded 111 CABSIs. We observed a progressive but significant decrease of CABSI rates from $1.48(95 \% \mathrm{Cl} 1.09-2.01)$ without CHG dressings to 0.69 (95\% Cl 0.43-1.09) and 0.23 (95\% Cl 0.11-0.48) episodes per 1000 catheter-days when CHG sponge and CHG gel dressings were used $(p=0.0007 ; p<0.001)$. A non-significant lower rate of infections occurred with CHG gel compared with CHG sponge dressings. An identical low rate of allergic skin reactions $(0.3 / 1000$ device-days) was observed with both types of CHX dressings. Post-study data until 2018 confirmed a sustained decrease of infection rates over 11 years.

Conclusions: The addition of chlorhexidine dressings to all CVC and arterial lines to an ongoing catheter bundle was associated with a sustained 11-year reduction of all catheter-associated bloodstream infections. This large real-world data study further supports the current recommendations for the systematic use of CHG dressings on all catheters of ICU patients.
\end{abstract}

Keywords: Catheter-related infections, Central line-associated bloodstream infections, Catheter bundle, Nosocomial infection, Bacteremia, Chlorhexidine-dressing, Chlorhexidine sponge, Chlorhexidine gel

\footnotetext{
*Correspondence: Philippe.eggimann@chuv.ch

${ }^{6}$ Department of Locomotor Apparatus, Centre Hospitalier Universitaire Vaudois (CHUV), Bugnon 46, BH 14-309, 1211 Lausanne, Switzerland

Full author information is available at the end of the article

Philippe Eggimann and Jean-Luc Pagani Equal contribution.
}

\section{垈




\section{Introduction}

Despite the successful widespread implementation of catheter bundles, central line-associated bloodstream infections remain an important cause of preventable nosocomial infections in patients requiring intensive care unit (ICU) management [1-4]. The multimodal strategies included in these bundles (i.e., standardization of procedures and care, skin preparation, hand hygiene, aseptic techniques and maximal barrier precaution for insertion) successfully addressed most recognized risk factors for catheter infections $[5,6]$. However, the efficacy of these recommended bundles seems to plateau around 1.0 episode per 1000 catheter-days [7-11]. This translates into the persistence of a significant number of preventable catheter-related infections in high-volume ICUs complying with the benchmark [12-14].

By controlling bacterial load at the site of insertion, dressings continuously releasing chlorhexidine gluconate ( $\mathrm{CHG}$ ) decreased the rate of central line-associated infections in two large prospective multicenter randomized trials among critically ill patients $[15$, 16]. These results were confirmed by a meta-analysis including nine randomized controlled trials comparing either CHG sponge or CHG gel dressings $(55,915$ catheter-days) with regular dressings ( 54,583 catheterdays) [17].

Based on these studies, recommendations for the use of CHG dressings have been included in the CDC and NHS guidelines [18, 19]. However, studies confirming the effectiveness of these antiseptic-containing dressings in a real-world setting are still lacking. Moreover, the two types of CHG dressings have never been directly compared.

We took advantage of a prospective bloodstream infection surveillance program for an ongoing catheter bundle to evaluate the impact of the progressive introduction of two types of CHG dressings on all arterial and central venous catheters inserted in patients admitted to a large mixed ICU over a 13-year period. For the first 2 years we collected baseline data, and during the following 7 years, we introduced the CHG dressings in four different steps. The objectives of the study were to assess whether the progressive introduction of CHG dressings to an ongoing catheter bundle could further reduce the rate of catheter-associated bloodstream infections and to compare the effectiveness of two different types of CHG dressing (CHG sponge + transparent dressing versus CHG gel all-inone dressing) on the rate of catheter-associated bloodstream infection.

\section{Take-home message}

The addition of chlorhexidine dressings to all CVC and arterial lines to an ongoing catheter bundle to all patients consecutively admitted to a large mixed ICU resulted in a significantly sustained 11-year reduction of all catheter-associated bloodstream infections

This large real-world data study further supports the current recommendations for the systematic use of CHG dressings on all catheters of ICU patients

\section{Methods \\ Design}

This is a real-word data study to evaluate the impact of CHG dressings in addition to an already ongoing catheter bundle on the incidence density rate of catheterassociated bloodstream infections (CABSIs).

\section{Setting}

A 35-bed mixed adult ICU of the Centre Hospitalier Universitaire Vaudois (CHUV), Lausanne, Switzerland, a primary and referral hospital for a population of 250,000 and 1,500,000, respectively. This is the only adult ICU in the hospital and consists of five distinct units. Allocation of patients is determined by bed availability and not by a patient's specific conditions.

\section{Participants}

All consecutive adult patients admitted to the ICU from January 2006 to December 2018 were included. Burn and ECMO patients were excluded from the analysis, as the enhanced bundle could not be systematically applied.

\section{Ethics}

As the interventions were included in the measures targeted at improvement of the quality of care, with products with a patent for use in patients in Switzerland, the commission cantonale d'ethique de la recherche sur l'être humain and the institutional review board approved the study and waived the need for informed consent.

\section{Data extraction}

As part of the routine and mandatory data collection to comply with the "minimal data set" of the Swiss Society of Intensive Care Medicine, demographic data and SAPS II scores were collected [20]. ICU and hospital length of stay and mortality were prospectively collected for each patient. Comorbidities were defined using definitions of the SAPS II score. 


\section{Intervention}

The intervention consisted of four stepwise modifications to an already established catheter bundle with progressive introduction of CHG dressings from 2007 to 2014 (Suppl Table 1).

\section{Comparator (baseline): period A (January 2006-October 2007)}

The standard of care as of January 2006 consisted of the following catheter bundle (Supplemental Table 2): (1) general infection control measures (hand hygiene, standard and isolation precautions); (2) guidelines for catheter insertion (checklist for material preparation, standardized hair removal with clippers and CHG-based skin antisepsis, maximal sterile barrier precaution); (3) handling (avoidance of needle and needleless connectors, systematic CHG disinfection of stop-cocks with new caps each time the hub is accessed); (4) maintenance (daily check for catheter necessity, signs of catheter infection, dressing integrity and need for replacement). To guarantee uniform application of the bundle, a designated physician and a designated nurse led the entire bundle and gave periodic feedback based on surveillance data [1, 7]. The designated clinical nurse specialist systematically trained ICU nurses on all elements of the bundle, including daily assessment of the dressings and face-to-face feedback and coaching. The designated physician systematically trained ICU physicians on how to apply the guidelines for insertion. This bundle was applied to all arterial and central venous lines [21].

\section{Stepwise interventions: periods B through E and post-study period}

To guarantee uniform implementation of the stepwise interventions (Supplemental Table 1), the dedicated clinical nurse specialist updated the institutional protocol, highlighted the changes specifically related to the use of the CHG dressings before each period, systematically trained ICU nurses to use these updated practices and completed a daily assessment of the CHG dressings with face-to-face feedback and coaching.

\section{Period B-November 2007-Nov 2009}

$\mathrm{CHG}$ sponge + transparent dressing on jugular and femoral CVC in all units.

\section{Period C-December 2009-May 2011}

$\mathrm{CHG}$ sponge + transparent dressing on all CVC and arterial catheters in all units.

Period D-June 2011-May 2013

CHG sponge + transparent dressing in three units (18 beds) and CHG gel all-in-one dressing in two units (14 beds) on all CVC and arterial catheters.
Period E-June 2013-December 2014

CHG gel all-in-one dressing in all units on all CVC and arterial lines.

Post-study period-January 2015-December 2018

CHG gel all-in-one dressing in all units on all CVC and arterial lines.

\section{Materials}

- CHG sponge dressing (Biopatch ${ }^{\circledR}$, Ethicon Inc., Somerville, NJ) covered by a transparent dressing $\left(3 \mathrm{M}^{\mathrm{TM}}\right.$ Tegaderm $^{\mathrm{TM}}$ without disinfectant, $3 \mathrm{M}$, Saint Paul, MN)

- CHG gel all-in-one dressing, containing CHG in a gel directly embedded within the transparent dressing (3 $\mathrm{M}^{\mathrm{TM}}$ Tegaderm $^{\mathrm{TM}}$ CHG Chlorhexidine Gluconate I.V. Securement Dressing, 3 M, Saint Paul, MN).

\section{Outcomes}

\section{Primary outcome}

Number of catheter-associated bloodstream infections (CABSIs) per 1000 catheter-days over each study period. Catheter-associated bloodstream infections were defined as either catheter-related infection or a primary bacteremia (Fig. 1). Secondary bloodstream infections were explicitly excluded [18].

\section{Secondary outcomes}

- Number of catheter-associated bloodstream infections per 1000 catheter-days by type of dressing and by type of catheter (CVC versus arterial).

- Numbers of catheter-related bloodstream infections (CRBSI) and primary bacteremia over each study period, by type of dressing and by type of catheter (CVC versus arterial).

\section{Measurements}

Measurement of catheter-associated bloodstream infections

A computer-based institutional surveillance program automatically identified every episode of positive blood culture linked to patients having stayed or actually staying in the ICU.

The hospital infection control staff reviewed each episode quarterly with the dedicated ICU physician. First, each episode was attributed to one the following categories: (1) community acquired ( $<48 \mathrm{~h}$ of hospital admission), (2) hospital non-ICU acquired ( $<48 \mathrm{~h}$ of ICU admission) and (3) hospital ICU acquired. Second, both community-acquired and hospital ICU-acquired positive blood cultures were further adjudicated to four categories: (1) catheter-related bloodstream infection, (2) primary bacteremia, (3) secondary bacteremia and (4) contamination [18]. 


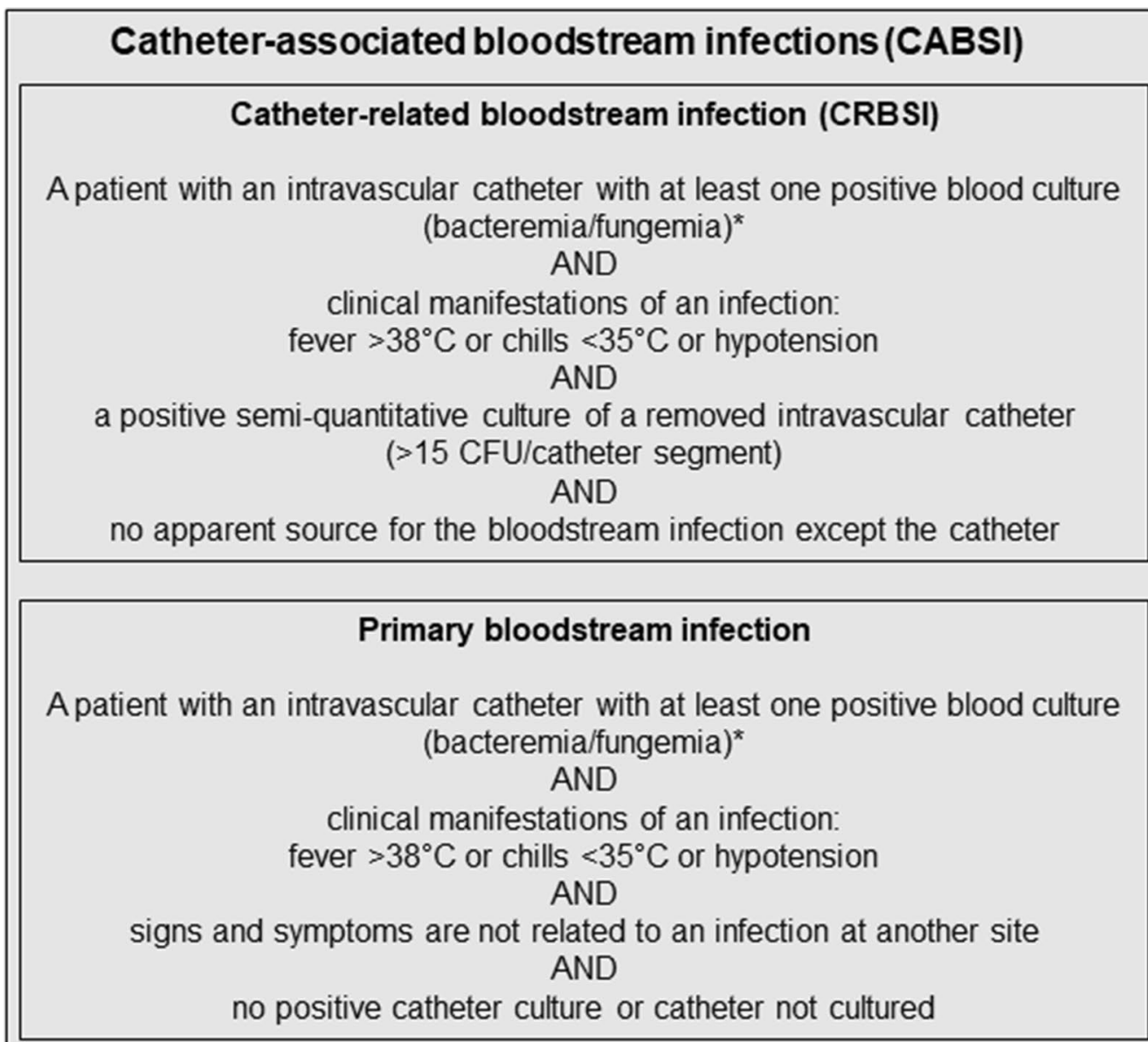

Secondary bloodstream infection
A patient with an intravascular catheter with at least one positive blood culture
(bacteremia/fungemia)*
AND
clinical manifestations of an infection:
fever $>38^{\circ} \mathrm{C}$ or chills $<35^{\circ} \mathrm{C}$ or hypotension
AND
signs and symptoms are related to an infection at another site than an
intravascular catheter

${ }^{\star}$ For common skin contaminant (e.g., diphtheroids, Bacillus spp., Propionibacterium spp., coagulasenegative staphylococci, or micrococci) positive culture is required from two or more blood cultures drawn on separate occasions.

Fig. 1 Definitions of infections. There are two different scenarios. Scenario 1: patient is admitted directly from outside of the hospital. In these cases, any episode of bloodstream infection was attributed to the ICU. Scenario 2: Patient is admitted from within the hospital. In this case, the episode of infection is attributed to the ICU only when it occurs $\geq 48 \mathrm{~h}$ from admission

\section{Measurement of catheter-days}

For each patient, the time of insertion of the catheter, time of its removal and catheter type (arterial-venous) were recorded in the computerized information system (Metavision, iMDsoft ${ }^{\circledR}$ Ltd., Tel Aviv Israel). For patients admitted with a catheter already inserted, the time of 
insertion was set as the time of patient ICU admission. Similarly, for patients discharged with a catheter in place, the time of discharge was set as equivalent to the time of catheter removal.

ICU catheter-days were computed as the sum of the catheter time duration (removal time minus insertion time) for every central venous catheter (including dialysis catheters and introducers sheaths for pulmonary artery catheter) and arterial catheters.

A new version of the computerized information system was implemented in January 2015. While this new version allows for a simplified computation of overall CVCdays, it precludes the analysis of data at the individual patient level. Meanwhile, other surveillance methods remain the same.

\section{Statistics}

\section{Demographics and patients characteristics}

Continuous variables are reported as the mean and standard deviation (SD) or as medians and interquartile ranges [IQR]. Categorical variables are reported as frequencies and percentages.

\section{Incidence density rates over the study periods}

The numbers of CABSI, CRBSI and primary bacteremia events per 1000 catheter-days over the different periods were reported as incidence density rates (and their 95\% $\mathrm{CI})$. The incidence density rates were tested using a Poisson regression model (SAS 9.3 PROC GENMOD). The $\log$ of the catheter-days was used as the offset, and the dispersion parameter was estimated to help determine whether over-dispersion needed to be accounted for in the Poisson model. Each period was compared with the baseline and the preceding one. In addition, period $C$, where only CHG sponge was used, was compared with period E, where only CHG gel was used. Multiple comparisons for the three measures of CABSI, CRBSI and primary bacteria were done using a Bonferroni correction $(p<0.05 \div 3=0.017$ was considered significant). Comparisons to period A were done using Dunnett's method.

During period D, CHG gel dressings were introduced in two units (14 beds), whereas CHG sponge dressings were maintained in the three other units (18 beds). This allowed for a contemporaneous comparison of the effect of both dressings using CABSI, CRBSI and primary bacteremia as outcomes. The comparisons were carried out using the same Poisson regression model.

\section{Sensitivity analysis}

Individual data and catheter-days were gathered from each individual ICU stay $(n=19,423)$ including the 111 CABSIs (70 CRBSIs and 41 primary bacteremia events).
We used a Cox proportional hazards regression model (SAS 9.3 PROC PHREG), with catheter-days representing the time factor and CABSI as the event of interest. Models were adjusted on age at ICU entry, SAPS 2 and duration of mechanical ventilation.

\section{Results}

\section{Characteristics of patients and risk factors for CABSI}

From January 2006 to December 2014, 18,286 patients were admitted to the medico-surgical ICU accounting for 91,292 treatment-days and 155,242 catheter-days consisting of 68,823 arterial and 89,419 central venous catheter-days. Most admissions were unscheduled ( $>70 \%)$, and $67 \%$ occurred after surgery (Table 1 ). Over time, the average age of patients went up; they were more severely affected (SAPS II) and required more organ failure support therapies such as mechanical ventilation or renal placement therapy. We observed an increase in catheterdays, case mix severity, workload as well as ICU and hospital mortalities over the study periods.

\section{Incidence density rates of CABSI}

Over the 9 years of observation, 111 CABSIs were diagnosed, including 70 CRBSI and 41 primary bacteremia cases (Supplemental Fig. 1). The stepwise introduction of various CHG dressings over the 9 years (supplemental Table 1) in addition to the ongoing catheter bundle (supplemental Table 2) progressively and significantly reduced CABSIs. Incidence density rates decreased from 1.48 (95\% CI 1.09-2.01) episodes per 1000 catheter-days in period $\mathrm{A}$ when no $\mathrm{CHG}$ dressings were used to 0.23 (95\% CI $0.11-0.48, p<0.001)$ episodes per 1000 catheterdays in period $\mathrm{E}$ when $\mathrm{CHG}$ gel dressings were used for all patients. Looking at the individual components of CABSI, the decrease in CRBSI from 1.12 (95\% CI 0.79$1.59)$ to $0.10(95 \% \mathrm{CI} 0.03-0.31, p<0.001)$ was statistically significant, but the decrease in primary bacteremia from 0.36 (95\% CI 0.19-0.67) to 0.13 (95\% CI 0.05-0.35, $p=0.059$ ) was not (Supplemental Fig. 2).

\section{Sensitivity analysis}

We performed a sensitivity analysis at the patient level to determine whether the variation in the case mix over the periods might have contributed to the observed reduction of the CABSI, CRBSI and primary bacteremia rates. After adjusting for age at ICU entry, SAPS 2 and duration of mechanical ventilation, the periods of bundle exposure still remained significant $(p<0.0001$, $p<0.0001$ and $p=0.013$ ), with periods $\mathrm{D}$ and $\mathrm{E}$ showing significantly lower CABSI rates than period A (baseline) $(p<0.0001, p<0.0001$ and $p=0.035)$, period $\mathrm{B}$ $(p<0.0001, p=0.0011$ and $p=0.014$, respectively) and period $C(p=0.0006, p=0.0024$ and $p=0.014)$. There 
Table 1 Characteristics of ICU activities and patients by study period

\begin{tabular}{|c|c|c|c|c|c|c|}
\hline \multirow[t]{2}{*}{ Period } & & A & B & C & D & 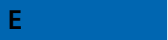 \\
\hline & All & $\begin{array}{l}\text { January } 2006 \\
\text { to October } \\
2007\end{array}$ & $\begin{array}{l}\text { November } 2007 \text { to } \\
\text { November } 2009\end{array}$ & $\begin{array}{l}\text { December } 2009 \text { to } \\
\text { May } 2011\end{array}$ & June 2011 to May 2013 & $\begin{array}{l}\text { June } 2013 \text { to } \\
\text { December } \\
2014\end{array}$ \\
\hline $\begin{array}{l}\text { Type of disinfectant } \\
\text { dressing added to } \\
\text { the ongoing catheter } \\
\text { bundle* }\end{array}$ & & None & $\begin{array}{l}\text { CHG sponge on CVC } \\
\text { only }\end{array}$ & $\begin{array}{l}\text { CHG sponge on all } \\
\text { catheters }\end{array}$ & $\begin{array}{l}\text { Replacement of CHG } \\
\text { sponge by CHG gel } \\
\text { in } 2 \text { out of } 5 \text { ICUs }\end{array}$ & $\begin{array}{l}\text { CHG gel on } \\
\text { all catheters } \\
\text { (five of five } \\
\text { ICUs) }\end{array}$ \\
\hline \multicolumn{7}{|l|}{ ICU activity } \\
\hline ICU treatment days & 91,292 & 17,185 & 20,429 & 15,476 & 20,726 & 17,476 \\
\hline $\begin{array}{l}\text { Mean ICU LOS, days } \\
\text { (SD) }\end{array}$ & $5.0(15.6)$ & $4.3(7.5)$ & $4.4(7.9)$ & $5.1(9.2)$ & $5.3(11.6)$ & $6.3(17.3)$ \\
\hline $\begin{array}{l}\text { Admissions (all ICU } \\
\text { stays), } n\end{array}$ & 19,423 & 4213 & 4931 & 3213 & 4133 & 2933 \\
\hline Emergency (\%) & $13,651(70.3)$ & $3037(72.1)$ & 3484 (71.5) & $2183(70.1)$ & $2872(71.3)$ & 2075 (70.7) \\
\hline $\begin{array}{l}\text { Surgical admissions, } \\
n(\%)\end{array}$ & $13,018(67.0)$ & $2779(66.0)$ & $3258(66.1)$ & 2265 (70.5) & $2749(66.5)$ & $1967(67.1)$ \\
\hline $\begin{array}{l}\text { Scheduled surgery, } \\
n(\%)\end{array}$ & $5722(29.5)$ & $1222(29.0)$ & 1409 (28.6) & $963(30.0)$ & $1211(29.3)$ & 917 (31.3) \\
\hline $\begin{array}{l}\text { Emergency surgery, } \\
n(\%)\end{array}$ & 7296 (37.5) & $1557(37.0)$ & $1849(37.5)$ & $1302(40.5)$ & $1538(37.2)$ & $1050(35.8)$ \\
\hline \multicolumn{7}{|l|}{ Site of surgery } \\
\hline Cardiovascular, n (\%) & $2131(16.4)$ & $436(15.7)$ & $536(16.5)$ & $343(15.1)$ & $452(16.4)$ & 364 (18.5) \\
\hline Digestive, $n(\%)$ & $1621(12.5)$ & $296(10.7)$ & $432(13.3)$ & $268(11.8)$ & $356(13.0)$ & $269(13.7)$ \\
\hline Neurosurgery, n (\%) & $1173(9.0)$ & $300(10.8)$ & $313(9.6)$ & $203(9.0)$ & $204(7.4)$ & $153(7.8)$ \\
\hline Thoracic, $n(\%)$ & $663(5.1)$ & $138(5.0)$ & $163(5.0)$ & $106(4.7)$ & $132(4.8)$ & $124(6.3)$ \\
\hline Trauma, n (\%) & 795 (5.9) & $174(6.3)$ & $194(6.0)$ & $137(6.1)$ & $145(5.3)$ & $145(7.4)$ \\
\hline $\begin{array}{l}\text { Workload: NEMS } \\
\text { mean (SD) per } 12 \mathrm{~h} \\
\text { shift }\end{array}$ & $30(25-37)$ & $28(27-32)$ & $31(27-37)$ & $31(27-37)$ & $30(25-37)$ & $29(22-36)$ \\
\hline \multicolumn{7}{|l|}{ ICU treatments } \\
\hline $\begin{array}{l}\text { Patients with ventila- } \\
\text { tion, admissions (\%) }\end{array}$ & $7146(36.8)$ & $1306(30.1)$ & $1533(31.1)$ & $1216(37.9)$ & $1492(36.1)$ & $1599(54.5)$ \\
\hline Ventilation-days & 50,852 & 8008 & 10,901 & 9445 & 12,574 & 9924 \\
\hline $\begin{array}{l}\text { Mechanical ventila- } \\
\text { tion, mean days/ } \\
\text { months (SD) }\end{array}$ & $471(108)$ & $364(76)$ & $436(80)$ & $525(77)$ & $524(116)$ & $522(79)$ \\
\hline Catheter-days & 155,242 & 27,713 & 34,363 & 26,128 & 36,689 & 30,349 \\
\hline CVC (\%) & $89,489(57.6)$ & $15,547(56.1)$ & 19,553 (56.9) & $15,024(57.5)$ & $21,641(59.0)$ & $17,724(58.4)$ \\
\hline CVC-jugular (\%) & $41,684(46.6)$ & $6618(42.6)$ & 8365 (42.8) & $7128(47.4)$ & $10,642(49.2)$ & 8931 (50.4) \\
\hline CVC-sub-clavian (\%) & $16,747(18.7)$ & 3549 (22.8) & $3740(19.1)$ & $3070(20.4)$ & $3866(17.9)$ & $2521(14.2)$ \\
\hline CVC-femoral (\%) & $31,058(34.7)$ & $5380(34.6)$ & $7448(38.1)$ & $4826(32.1)$ & $7132(33.0)$ & $6272(35.4)$ \\
\hline Arterial (\%) & $65,753(42.3)$ & $12,166(43.9)$ & $14,810(43.1)$ & $11,104(42.5)$ & $15,048(41.0)$ & $12,625(41.6)$ \\
\hline $\begin{array}{l}\text { Catheter-days per } \\
\text { month, mean (SD) }\end{array}$ & $1419(275)$ & $1161(189)$ & $1322(191)$ & $1471(169)$ & $1545(295)$ & $1636(232)$ \\
\hline $\begin{array}{l}\text { Patient with extra- } \\
\text { renal replacement } \\
\text { therapy, admissions, } \\
(\%)\end{array}$ & $1621(8.4)$ & $204(4.8)$ & $352(7.1)$ & $250(7.8)$ & $424(10.3)$ & $391(13.3)$ \\
\hline $\begin{array}{l}\text { Mean extra-renal } \\
\text { replacement } \\
\text { therapy, mean } \\
\text { hours/month (SD) }\end{array}$ & 1370 (847) & $763(460)$ & $1162(717)$ & $1037(437)$ & $1824(883)$ & 2091 (843) \\
\hline \multicolumn{7}{|l|}{ Patients } \\
\hline Number & 18,286 & 3981 & 4607 & 3022 & 3901 & 2775 \\
\hline
\end{tabular}


Table 1 (continued)

\begin{tabular}{|c|c|c|c|c|c|c|}
\hline Period & & A & B & C & D & E \\
\hline $\begin{array}{l}\text { Age, year (median, } \\
\text { IQR) }\end{array}$ & 63.7 (51.4-73.9) & $62.6(50.3-73.2)$ & $63.6(50.8-74.4)$ & $65.0(54.3-74.5)$ & $64.3(52.3-74.6)$ & $\begin{array}{l}65.2(52.6- \\
74.5)\end{array}$ \\
\hline Males, $n(\%)$ & $12,025(65.8)$ & $2632(66.1)$ & $3040(66.0)$ & $1995(66.0)$ & $2536(65.0)$ & $1822(65.7)$ \\
\hline \multicolumn{7}{|l|}{ Patient comorbidities } \\
\hline NYHA IV (\%) & $1864(10.2)$ & $396(9.9)$ & $554(12.0)$ & $348(11.5)$ & 335 (8.6) & $231(8.3)$ \\
\hline Chronic dialysis, $n(\%)$ & $396(2.2)$ & $80(2.0)$ & $114(2.5)$ & $49(1.6)$ & $67(1.7)$ & $86(3.1)$ \\
\hline Severe COPD, $n(\%)$ & $1383(7.6)$ & $309(7.8)$ & $312(6.8)$ & $215(7.1)$ & $288(7.4)$ & $259(9.3)$ \\
\hline Liver cirrhosis, n (\%) & $739(4.0)$ & $128(3.2)$ & $171(3.7)$ & $121(4.0)$ & $182(4.7)$ & $137(4.9)$ \\
\hline $\begin{array}{l}\text { Metastatic cancer, } \\
n(\%)\end{array}$ & $2356(12.9)$ & 461 (11.6) & $581(12.6)$ & $375(12.4)$ & $541(13.9)$ & $398(14.3)$ \\
\hline $\begin{array}{l}\text { Mc Cabe-within } \\
5 \text { years, } n(\%)\end{array}$ & $5088(27.8)$ & $1078(27.1)$ & $1342(29.1)$ & $824(27.3)$ & $1021(26.2)$ & $823(29.7)$ \\
\hline $\begin{array}{l}\text { Mc Cabe-within } \\
1 \text { year, } n(\%)\end{array}$ & $1741(9.5)$ & $290(7.3)$ & $415(9.0)$ & $265(8.8)$ & $458(11.7)$ & $313(11.3)$ \\
\hline \multicolumn{7}{|l|}{ Major critical illness } \\
\hline $\begin{array}{l}\text { Hemorrhagic shock, } \\
n(\%)\end{array}$ & 657 (3.6) & $130(3.3)$ & $100(2.2)$ & $112(3.7)$ & $166(4.3)$ & $149(5.4)$ \\
\hline $\begin{array}{l}\text { Cardiogenic shock, } \\
n(\%)\end{array}$ & $726(4.0)$ & $166(4.2)$ & $157(3.4)$ & $126(4.2)$ & $136(3.5)$ & $141(5.1)$ \\
\hline Septic shock, n (\%) & $1299(7.1)$ & $207(5.2)$ & $326(7.1)$ & $180(6.0)$ & $331(8.5)$ & $255(9.2)$ \\
\hline Pneumonia, $n$ (\%) & $2511(13.7)$ & $544(13.7)$ & $587(12.7)$ & $382(12.6)$ & $544(13.9)$ & $454(16.4)$ \\
\hline Peritonitis, $n(\%)$ & $408(2.2)$ & $87(2.2)$ & $114(2.5)$ & $58(1.9)$ & $81(2.1)$ & $68(2.5)$ \\
\hline \multicolumn{7}{|l|}{ Severity } \\
\hline SAPS II median (IQR) & $38(27-49)$ & $35(25-45)$ & $36(26-47)$ & $38(29-49)$ & $41(30-53)$ & $40(28-51)$ \\
\hline ICU mortality, n (\%) & 1974 (10.8) & $386(9.7)$ & $441(9.6)$ & $305(10.1)$ & $474(12.2)$ & $368(13.3)$ \\
\hline $\begin{array}{l}\text { Hospital post-ICU } \\
\text { mortality, } n(\%)\end{array}$ & $539(2.9)$ & $115(2.9)$ & $157(3.4)$ & $100(3.3)$ & $99(2.5)$ & $68(2.5)$ \\
\hline
\end{tabular}

CRBSI catheter-related bloodstream infection, $C A B S I$ catheter-associated bloodstream infection, i.e., CRBSI + primary bacteremia

*See supplemental Table 2

were no significant differences between period $\mathrm{D}$ and $\mathrm{E}$ $(p=0.96$ and $p<0.08)$. Hence, these results confirm the overall findings of the main analysis.

\section{Comparison of CHG dressings}

We sequentially introduced two types of CHG dressings for all catheters: $\mathrm{CHG}$ sponge over periods $\mathrm{B}$ and $\mathrm{C}$ and CHG gel dressings over periods D and E. During period D, CHG gel replaced CHG sponge in two of the five ICU units. We therefore also compared period C (CHG sponge on all catheters) with period E (CHG gel on all catheters) in Table 2. We observed a decrease of CABSI when CHG gel dressing (7 episodes) was used instead of CHG sponge dressing (18 episodes), translating into a reduction of incidence density rates to 0.23 episodes per 1000 catheter-days (95\% CI 0.11-0.48) in period E compared with 0.69 (95\% CI $0.43-1.09, p=0.019)$ in period $\mathrm{C}$. The $p$ values for CRBSI and primary bacteremia were 0.018 and 0.99 , respectively (Table $2 \mathrm{a}$ ). Period D was the time when both CHG sponge and CHG gel were used, providing another opportunity to compare the dressings
(Table 2b). During period D, we observed nine infections (0.43 CABSI per 1000 catheter-days; 95\% CI 0.23-0.83) with CHG sponge compared with two (0.13 CABSI per 1000 catheter-days; 95\% CI 0.03-0.50) with CHG gel. The concomitant comparison did not, however, reach statistical significance $(p=0.059$, Table $2 b)$.

\section{Post-study period}

Data from January 2015 to December 2018 showed a CABSI rate of 0.32 (95\% CI 018-0.49) episodes per 1000 central venous catheter-days, suggesting that the progressive decrease we observed during the study was sustained post-study (Fig. 2).

\section{Adverse effect of CHG dressings}

We evaluated skin tolerance during the introduction of Tegaderm ${ }^{\mathrm{TM}}$ CHG during period D. To this end, an independent dermatologist assessed all the observed skin reactions (Supplemental Fig. 3). From June 2011 to January 2012, 26 skin reactions were recorded with Tegaderm $^{\text {TM }}$ CHG (5.5 episodes/1000 device-days) and 
Table 2 Comparison of two types of CHG dressings

\begin{tabular}{|c|c|c|c|c|}
\hline \multicolumn{5}{|c|}{ (a) Period C (sponge only) versus period E (gel only) } \\
\hline & All & Period C sponge only & Period E gel only & $\begin{array}{l}\text { Period E vs. } \\
\text { period C }\end{array}$ \\
\hline Catheter-day & 56,477 & 26,128 & 30,349 & \\
\hline CABSI & 25 & 18 & 7 & \\
\hline Incidence density rate of infection ( $95 \% \mathrm{Cl}$ ) & $0.44(0.28-0.66)$ & $0.69(0.43-1.09)$ & $0.23(0.11-0.48)$ & 0.019 \\
\hline CRBSI & 14 & 11 & 3 & \\
\hline Incidence density rate of infection $(95 \% \mathrm{Cl})$ & $0.25(0.14-0.42)$ & $0.42(0.23-0.76)$ & $0.10(0.03-0.31)$ & 0.018 \\
\hline Primary bacteremia & 11 & 7 & 4 & \\
\hline Incidence density rate of infection ( $95 \% \mathrm{Cl}$ ) & $0.19(0.10-0.35)$ & $0.27(0.13-0.56)$ & $0.13(0.05-0.35)$ & 0.99 \\
\hline \multicolumn{5}{|c|}{ (b) CHG sponge vs. CHG gel (intervention 3, period D) } \\
\hline Period D only June 2011 to May 2013 & All & CHG sponge 18 beds & CHG gel 14 beds & $\begin{array}{l}\text { Comparison } \\
\text { of gel ver- } \\
\text { sus sponge }\end{array}$ \\
\hline Catheter-day & 36,689 & 20,807 & 15,882 & \\
\hline CABSI & 11 & 9 & 2 & \\
\hline Incidence density rate of infection ( $95 \% \mathrm{Cl}$ ) & $0.30(0.17-0.54)$ & $0.43(0.23-0.83)$ & $0.13(0.03-0.50)$ & 0.059 \\
\hline CRBSI & 6 & 4 & 2 & \\
\hline Incidence density rate of infection ( $95 \% \mathrm{Cl}$ ) & $0.16(0.07-0.36)$ & $0.19(0.07-0.51)$ & $0.13(0.03-0.50)$ & 0.49 \\
\hline Primary bacteremia & 5 & 5 & 0 & \\
\hline Incidence density rate of infection ( $95 \% \mathrm{Cl}$ ) & $0.14(0.06-0.33)$ & $0.24(0.10-0.58)$ & Undefined & $<0.001$ \\
\hline
\end{tabular}

Infections ( $n$ ) and incidence density rates of infections (episodes per 1000 catheter-days; $95 \% \mathrm{Cl}$ ) by type of dressing

5 with Biopatch $^{\circledR}$ (0.9 episodes/1000 device-days). They were all scored as allergic reactions. In March 2012, we introduced a newly designed Tegaderm ${ }^{\text {TM }}$ CHG with improved evaporation capability, while the Biopatch $^{\circledR}$ did not change. From March 2012 to May 2013, two skin reactions were observed with Tegaderm ${ }^{\text {TM }}$ CHG $(0.3 / 1000$ device-days) and one with Biopatch ${ }^{\circledR}$ (0.3/1000 devicedays). Of note, no CABSIs occurred in these patients.

\section{Discussion}

The stepwise introduction of two types of CHG dressings over a 7-year period achieved a sharp and sustained reduction in all types of catheter-related infections, despite increased workload, catheter use and severity of illness.

For the first time, a real-world data study, consisting of prospective surveillance of 155,242 catheter-days, confirms that the addition of CHG dressings to existing catheter bundles provides a significant decrease in the rate of catheter-related bloodstream infections in a real-world setting. Post-study surveillance data showed a persistent low rate of infections over an additional 4-year period.

CRBSI are considered the most preventable nosocomial infection [4]. Over the last 2 decades, catheter bundles, aiming at reducing contamination during insertion procedures and subsequent hub handling, resulted in a substantial decrease in CLABSI rates, down to values between one and two episodes per 1000 catheter-days [3, $12,14]$.

By adding an antimicrobial agent to control the bacterial load at the insertion site, chlorhexidine dressings can further reduce the CLABSI rate. Timsit et al. conducted two milestone prospective randomized controlled trials on this topic. The first study [15] tested the same CHG sponge dressing we used in periods $\mathrm{B}, \mathrm{C}$ and $\mathrm{D}$. It evaluated 1636 patients for a total of 28,931 catheter-days. Compared with standard dressings, the CHG sponge dressings achieved a decrease in catheter-related infections (defined as either catheter-related clinical sepsis without bloodstream infection or CRBSI) from 1.4 to 0.6 episodes per 1000 catheter-days (hazard ratio: 0.39; 95\% CI $0.17-0.93 ; p=0.03)$. The second study [16] tested the same CHG gel dressing we used in periods D and E. It evaluated 1879 patients for a total of 34,339 catheterdays. Compared with non-chlorhexidine dressings, the CHG gel dressings achieved a decrease in CRI (defined as in the previous study) from 2.1 to 0.7 episodes per 1000 catheter-days (hazard ratio: 0.328 ; 95\% CI 0.174-0.619; $p=0.0006$ ) and a decrease in CRBSI from 1.3 to 0.5 episodes per 1000 catheter-days (hazard ratio: 0.402 ; $95 \% \mathrm{CI}$ $0.186-0.868 ; p=0.02$ ).

Our data not only confirm these results in a real-word setting, but also provide additional evidence to support the recommendation endorsed by the guidelines for the 


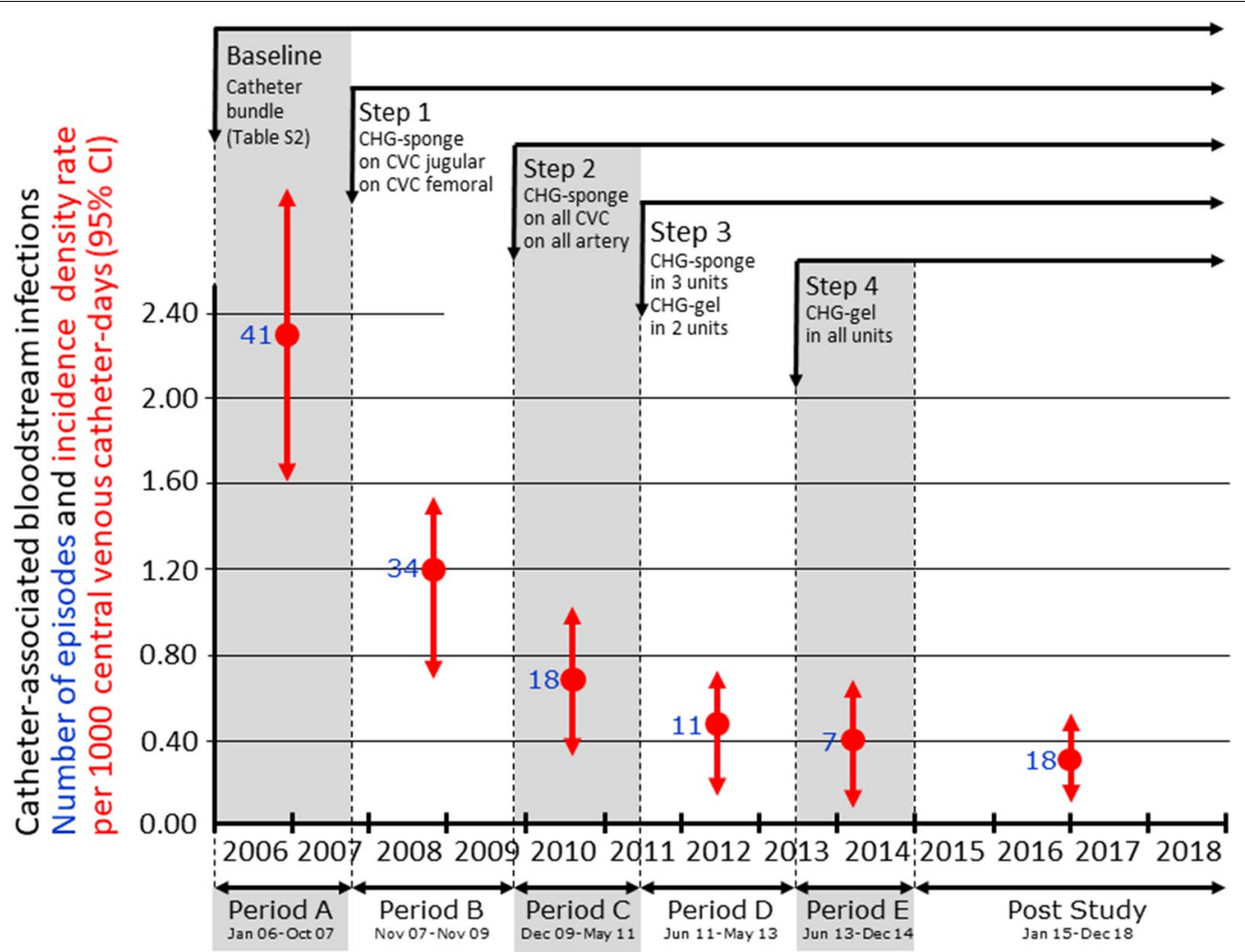

Fig. 2 Post-study surveillance data (2015-2018) of catheter-associated bloodstream infections (CABSI). CABSI expressed as rate of infection by central venous catheter-days. Period A: 2.31 (95\% Cl 1.61-3.0) CABSIs per 1000 central venous catheter-days; period B: 1.15 (0.77-1.54); period C: 0.69 (0.37-1.01); period D: 0.47 (0.19-0.75); period E: 0.38 (0.10-0.67); post-study period: $0.34(0.18-0.49)$

generalized use of CHG dressings in acutely ill patients $[18,19]$. Comparing the initial period, during which the state-of-the-art bundle was in use, and the last period during which the CHG gel dressing was used for all patients and catheters, we observed a sixfold reduction in the CABSI rate, corresponding to 16 (95\% CI 13.6-19.3) episodes avoided every year.

Moreover, the enhanced bundle impacted CRBSI and primary bacteremia similarly, independently of case mix variation. This suggests that primary bacteremia might be correlated with catheter infection in situations such as ours where catheter tip cultures are not systematically performed.

Our study has several strengths. First, it is a large realworld data study including $>18,000$ patients and 160,000 catheter-days over 9 years. This is comparable to the recent meta-analysis including nine studies with antiseptic dressings [17]. Second, the observation was done in a mixed tertiary ICU admission with severely critically ill patients, as assessed by high severity scores and workload required to manage them. The single-center design limits the external validity on one hand but ensures a higher degree of consistency of processes of care on the other. Third, the sustained decrease in infection rate was achieved despite a continuous increase in case mix severity over time. Finally, the recording of the data was performed without changes in the setting of the surveillance, in the definitions of infections or in the staff in charge of catheter-bundle training and maintenance.

Our study also has several limitations. First, it is monocentric and without randomization and therefore does not qualify for a pragmatic trial. Hence, we cannot discriminate between the relative impact of the ongoing bundle over time and the precise contribution of CHG dressings. However, data from the literature suggest that if catheter bundles have a sustainable effect, this seems to be limited to stabilization of the rate of infection rather than permitting a continuous further decrease over time [7, 8, 22, 23]. Second, the study design did not allow determining the effect of the generalization of alcoholic $2 \%$ chlorhexidine for skin preparation introduced in period D. Third, although systematic education and continuous bedside teaching to comply with the catheter bundle were provided, we never formally assessed compliance. Fourth, the absence of systematic catheter culture may result in an underestimation of the true rate of 
CRBSI. Episodes may have been scored as primary bacteremia and then included in CABSI already defined as the primary end point. Finally, the improved results might result not only from the use of chlorhexidine dressings on top of the bundle, but also from the creation of an environment and culture of quality improvement commitment among staff, generated by the long-lasting chain of quality improvement initiatives, with breakthrough interventions, supported by educational tools and data feedback.

Our data suggest that CHG gel may be more effective than CHG sponge to prevent CABSI in adult patients requiring ICU management. Comparing periods $\mathrm{C}$ (CHG sponge on all catheters) and $\mathrm{E}$ (CHG gel on all catheters), the CABSI and CRBSI incidence density rates almost reached the significance level of 0.017 accounting for multiple comparisons ( $p=0.019$ and 0.018 , respectively; see Table 2a). Due to the small numbers of infections, the power to detect a difference between those periods was only $77 \%$ and not the usual $80 \%$ targeted. For period D during which both dressings were used concomitantly and where the effect of the ongoing catheter bundle was identical, the difference did not reach significance either (two episodes, hazard ratio: 0.13; 95\% CI 0.03-0.50, versus nine episodes, hazard ratio: 0.43 ; $95 \%$ CI $0.23-0.83$, per 1000 catheter-days, $p=0.059)$. The absence of significant difference may be due to low power to assess a significant difference in this comparison (49\%) or to random chance. A potential significant superiority of the gel could further improve infection control and decrease rates of CABSI. This hypothesis should however be addressed by a sufficiently powered prospective controlled study.

Calculation of cost-effectiveness was beyond the scope of the study. However, the stepwise implementation of the enhanced catheter bundle has changed the empirical management of patients developing sepsis of unknown origin. First, systematic catheter change (guidewire exchange or insertion at new sites) was no longer required, thus avoiding the risk associated with new catheterization. Second, for the same reason, vancomycin was not systematically added in empirical antibiotic treatment of sepsis of unknown origin to treat an eventual CABSI, thus contributing to reducing antibiotic exposure. Finally, these changes in patient management combined with a sustained reduction in CABSI might have resulted in cost reduction as suggested by Maunoury et al. [5, 24].

We conclude that the addition of chlorhexidine dressings to all CVC and arterial lines to an ongoing catheter bundle in all patients requiring ICU admission resulted in a sustained and probably cost-effective 11 -year reduction of catheter-associated bloodstream infections.

\section{Electronic supplementary material}

The online version of this article (https://doi.org/10.1007/s00134-019-05617-x) contains supplementary material, which is available to authorized users.

\begin{abstract}
Author details
${ }^{1}$ Service of Adult Intensive Care Medicine, University Hospital of Lausanne, University of Lausanne, Lausanne, Switzerland. ${ }^{2}$ Unit of Population Epidemiology, Department of Community Medicine, Primary Care and Emergency Medicine, Geneva University Hospitals, Geneva, Switzerland. ${ }^{3} 3 \mathrm{M}$ Health Care Business, 3 M Center, St. Paul, MN, USA. ${ }^{4}$ Service of Hospital Preventive Medicine, University Hospital of Lausanne, University of Lausanne, Lausanne, Switzerland. ${ }^{5}$ Department of Intensive Care Medicine, Inselspital, Bern University Hospital, University of Bern, Bern, Switzerland. ${ }^{6}$ Department of Locomotor Apparatus, Centre Hospitalier Universitaire Vaudois (CHUV), Bugnon 46, BH 14-309, 1211 Lausanne, Switzerland.
\end{abstract}

\section{Acknowledgements}

We thank the medical and nursing team of the Adult Mixed ICU from the Centre Hospitalier Universitaire Vaudois for their cooperation with our protocol and for data collection; we thank Stéphanie F. Bernatchez from 3M for writing assistance and language editing and Laurent Seravalli, MD, for pertinent comments along the intervention; we thank François Eggimann, data manager for the extraction of data from the datawarehouse computerized information system.

\section{Author contribution}

$P E, J L P, J P R$ and $Y A Q$ were involved in the conception and the design of the intervention, analyzed the data and wrote the paper. EDL and BE performed the statistical work. PE, JLP, JPR, YAQ and MJT collected the data on bacteremia. MJT, PE, JLP, JPR and YAQ prospectively reviewed all episodes of bacteremia. PE, JLP, JPR, YAQ and $C J$ instructed medical doctors and nursing personnel for the component of the intervention SFB provided writing assistance and language editing. All authors reviewed the paper and agreed with final version of the manuscript.

\section{Funding}

Partially supported by an unrestricted research grant from 3M.

\section{Compliance with ethical standards}

\section{Conflicts of interest}

$\mathrm{PE}$ and $\mathrm{CJ}$ served on the speaker's bureau of $3 \mathrm{M}$ and of Ethicon, manufacturer of the disinfectant dressings used during the intervention. PE received an unrestricted research grant to support the analysis of the data. BE from $3 \mathrm{M}$ performed the statistical work. SFB from $3 \mathrm{M}$ provided writing assistance and language editing. Part of the data were provided to $3 \mathrm{M}$ and to the FDA in March 2017. The authors declared no other conflicts of interest.

\section{Open Access}

This article is distributed under the terms of the Creative Commons Attribution-NonCommercial 4.0 International License (http://creativecommons.org/ licenses/by-nc/4.0/), which permits any noncommercial use, distribution, and reproduction in any medium, provided you give appropriate credit to the original author(s) and the source, provide a link to the Creative Commons license, and indicate if changes were made.

\section{Publisher's Note}

Springer Nature remains neutral with regard to jurisdictional claims in published maps and institutional affiliations.

Received: 12 November 2018 Accepted: 6 April 2019

Published online: 17 April 2019

\section{References}

1. Eggimann P, Harbarth S, Constantin MN, Touveneau S, Chevrolet JC, Pittet D (2000) Impact of a prevention strategy targeted at vascular-access 
care on incidence of infections acquired in intensive care. Lancet 355:1864-1868

2. Pronovost P, Needham D, Berenholtz S et al (2006) An intervention to decrease catheter-related bloodstream infections in the ICU. N Engl J Med 355:2725-2732

3. Ista E, van der Hoven B, Kornelisse RF et al (2016) Effectiveness of insertion and maintenance bundles to prevent central-line-associated bloodstream infections in critically ill patients of all ages: a systematic review and meta-analysis. Lancet Infect Dis 16:724-734

4. Lambert ML, Silversmit G, Savey A et al (2014) Preventable proportion of severe infections acquired in intensive care units: case-mix adjusted estimations from patient-based surveillance data. Infect Control Hosp Epidemiol 35:494-501

5. Timsit JF, L'Heriteau F, Lepape A et al (2012) A multicentre analysis of catheter-related infection based on a hierarchical model. Intensive Care Med 38:1662-1672

6. Haga Y, Miyanari N, Takahashi T et al (2013) Risk factors for catheterrelated bloodstream infections in adult hospitalized patients - multicenter cohort study. Scand J Infect Dis 45:773-779

7. Eggimann P, Hugonnet S, Sax H, Harbarth S, Chevrolet JC, Pittet D (2005) Long-term reduction of vascular access-associated bloodstream infection. Ann Intern Med 142:875-876

8. Pronovost PJ, Watson SR, Goeschel CA, Hyzy RC, Berenholtz SM (2016) Sustaining reductions in central line-associated bloodstream infections in Michigan intensive care units: a 10-year analysis. Am J Med Qual 31:197-202

9. Lin WP, Chang YC, Wu UI et al (2018) Multimodal interventions for bundle implementation to decrease central line-associated bloodstream infections in adult intensive care units in a teaching hospital in Taiwan, 20092013. J Microbiol Immunol Infect 51:644-651

10. Castagna HM, Kawagoe JY, Goncalves P et al (2016) Active surveillance and safety organizational goals to reduce central line-associated bloodstream infections outside the intensive care unit: 9 years of experience. Am J Infect Control 44:1058-1060

11. Lee GM, Kleinman K, Soumerai SB et al (2012) Effect of nonpayment for preventable infections in US hospitals. N Engl J Med 367:1428-1437

12. Furuya EY, Dick A, Perencevich EN, Pogorzelska M, Goldmann D, Stone PW (2011) Central line bundle implementation in US intensive care units and impact on bloodstream infections. PLoS One 6:e15452

13. Ziegler MJ, Pellegrini DC, Safdar N (2015) Attributable mortality of central line associated bloodstream infection: systematic review and metaanalysis. Infection 43:29-36
14. van der Kooi T, Sax H, Pittet D et al (2018) Prevention of hospital infections by intervention and training (PROHIBIT): results of a pan-European cluster-randomized multicentre study to reduce central venous catheterrelated bloodstream infections. Intensive Care Med 44:48-60

15. Timsit JF, Schwebel C, Bouadma L et al (2009) Chlorhexidine-impregnated sponges and less frequent dressing changes for prevention of catheter-related infections in critically ill adults: a randomized controlled trial. JAMA 301:1231-1241

16. Timsit JF, Mimoz O, Mourvillier B et al (2012) Randomized controlled trial of chlorhexidine dressing and highly adhesive dressing for preventing catheter-related infections in critically ill adults. Am J Respir Crit Care Med 186:1272-1278

17. Safdar N, O'Horo JC, Ghufran A et al (2014) Chlorhexidine-impregnated dressing for prevention of catheter-related bloodstream infection: a meta-analysis*. Crit Care Med 42:1703-1713

18. O'Grady NP, Alexander M, Dellinger EP et al (2002) Guidelines for the prevention of intravascular catheter-related infections. Centers for Disease Control and Prevention. MMWR Recomm Rep 51:1-29

19. Jenks M, Craig J, Green W, Hewitt N, Arber M, Sims A (2016) Tegaderm CHG IV securement dressing for central venous and arterial catheter insertion sites: a NICE medical technology guidance. Appl Health Econ Health Policy 14:135-149

20. Le Gall JR, Lemeshow S, Saulnier F (1993) A new simplified acute physiology score (SAPS II) based on a European/North American multicenter study. JAMA 270:2957-2963

21. Lucet JC, Bouadma L, Zahar JR et al (2010) Infectious risk associated with arterial catheters compared with central venous catheters. Crit Care Med 38:1030-1035

22. Bion J, Richardson A, Hibbert P et al (2013) 'Matching Michigan': a 2-year stepped interventional programme to minimise central venous catheterblood stream infections in intensive care units in England. BMJ Qual Saf 22:110-123

23. Palomar M, Alvarez-Lerma F, Riera A et al (2013) Impact of a national multimodal intervention to prevent catheter-related bloodstream infection in the ICU: the Spanish experience. Crit Care Med 41:2364-2372

24. Maunoury F, Farinetto C, Ruckly S et al (2018) Cost-effectiveness analysis of a transparentvantimicrobial dressing for managing central. Venous and arterial catheters in intensiveve care units. PLoS One. 13(5):e0197747 